\title{
Effect of modulation amplitude on low-field microwave absorption in hafnium-doped $\mathrm{Y}-\mathrm{Ba}-\mathrm{Cu}-\mathrm{O}$
}

\author{
Md SHAHABUDDIN, A G VEDESHWAR, H D BIST ${ }^{+}$, PREM $^{2}$ \\ CHAND, S K AGARWAL* and A V NARLIKAR* \\ Department of Physics, Indian Institute of Technology, Kanpur 208 016, India \\ * National Physical Laboratory, New Delhi 110012, India.
}

\begin{abstract}
Low-field differential microwave absorption has been studied for hafnium-doped $\mathrm{Y}-\mathrm{Ba}-\mathrm{Cu}-\mathrm{O}$ at the first harmonic of modulation field. The effect of modulation amplitude on peak height, peak position and the area under hysteresis curve has been discussed.
\end{abstract}

Keywords. Microwave absorption; Hf doping; modulation amplitude.

\section{Introduction}

The recently discovered high $T_{c}$ superconducting (HTSC) materials provide puzzles, both on microscopic and macroscopic levels. At the microscopic level it is the mechanism for pairing that is being debated. At the more phenomenological level it is the apparent glass-like properties of the materials (Mullar et al 1987) or the giant flux creep (Yeshurun and Malozemoff 1988) that is drawing experimental and theoretical attention. The non-resonant low field microwave absorption (LFMAS) in HTSC is interesting. The imaginary parts of the resultant field-dependent susceptibility gives rise to large absorption in low magnetic fields. Most of the properties of the LFMAS have been explained in terms of the intergrain Josephson currents between weakly coupled clusters of superconducting grains (Blazey et al 1987; Khachaturyan et al 1987).

The EPR spectrometer yields the first derivative of the actual absorption line when small modulation amplitude is used. The modulation effect on LFMAS in $\mathrm{YBa}_{2} \mathrm{Cu}_{3} \mathrm{O}_{6.93}$ has been studied (Rakvin et al 1989) and the shape of the LFMAS detected at first and second harmonic had been explained using the earlier developed relation between surface impedance and magnetic field (Portis et al 1988) and introducing of weak modulation field into surface impedance.

We report the effect of modulation amplitude on the peak height $\left(h_{p}\right)$, peak position $\left(H_{m}\right)$ and the area under the hysteresis curve $(\Delta A)$ of LFMAS.

\section{Experimental}

Hafnium doped $\mathrm{Y}-\mathrm{Ba}-\mathrm{Cu}-\mathrm{O}$ samples were prepared in the same way as in the case of $\mathrm{Zr}$-substituted samples using solid-state mixing technique reported elsewhere (Jayaram et al 1988). Materials were characterised by XRD (Sieman D-500 X-ray diffractometer) and SEM (JEOL JSM 3SCF scanning microscope). Resistance was

\footnotetext{
${ }^{\dagger}$ For Correspondance
} 


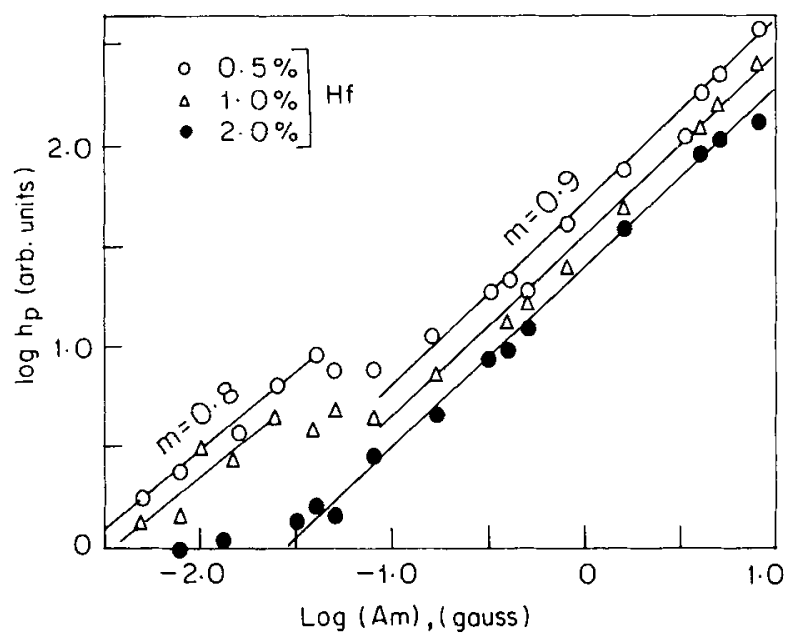

Figure 1. Log-log plot of $h_{p}$ vs $A_{m}$ for Hf-doped $\mathrm{Y}-\mathrm{Ba}-\mathrm{Cu}-\mathrm{O}$ samples. Slopes are specified at each plot.

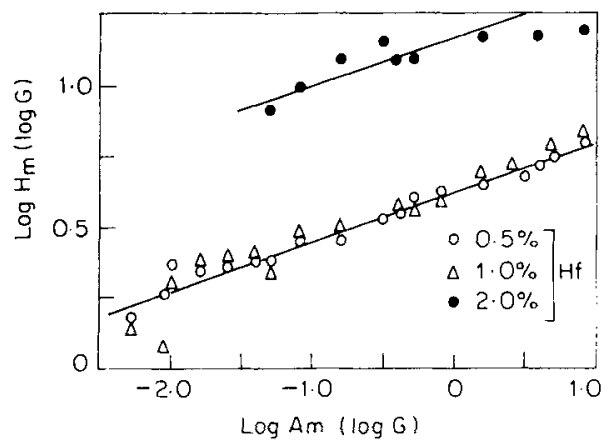

Figure 2. Log-log plot of $H_{m}$ vs $A_{m}$ for different Hf-doped samples.

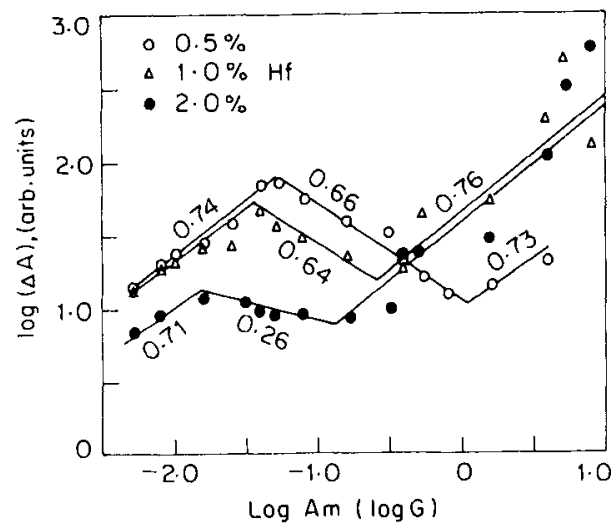

Figure 3. Log-log plot of the area under hysteresis loop $\triangle A$ vs $A_{m}$ for different Hf-doped $\mathrm{Y}-\mathrm{Ba}-\mathrm{Cu}-\mathrm{O}$ samples. Slopes are specified at each plot in all regions. 
measured by the conventional four-probe technique and the temperature measured by a standard $100 \mathrm{ohm}$ platinum resistance thermometer. For studies of the modulation field amplitude effect on low field microwave absorption signal (LFMAS) an $100 \mathrm{kHz}$ phase sensitive detection has been used and first derivative of absorption signal was recorded at $\mathrm{X}$-band microwave frequencies $(\sim 9 \mathrm{GHz})$. Samples in equal amount $(\sim 20 \mathrm{mg}$ ) were taken in spherical shape from the pellets and protected from humidity by sealing them in quartz tubes. LFMAS were recorded at liquid nitrogen temperature by dipping directly the thin sealed quartz tube containing the sample in liquid nitrogen. A varian E-109 X band spectrometer was used to record the LFMAS. A pair of Helmholtz coil and current source was used to cancel the residual magnetic field of the electromagnet and to provide the scan through zero field.

\section{Results}

The three samples studied have $0.5 \%, 1 \%$ and $2 \%$ Hf doping. The zero resistance transition temperature $T_{\mathrm{c}}$ was 90,89 and $88 \mathrm{~K}$ for $0.5 \%, 1 \%$ and $2 \% \mathrm{Hf}$-doped samples respectively.

The LFMAS for each of the samples was recorded keeping microwave power, temperature and modulation frequency constant in the following steps (i) The sample was zero field cooled to LNT and the signal in the forward direction was recorded, followed by a reverse sweep recording back to zero field, (ii) the first step was repeated changing the modulation field amplitude.

LFMAS is sensitive to modulation amplitude. Parameters like $h_{p}, H_{m}$ and $\Delta A$ of LFMAS are sensitive to modulation amplitude. The $h_{p}$ for all three samples as a function of modulation amplitude $\left(A_{m}\right)$ is shown in figure 1 . It is clear that $h_{p}$ increases approximately linearly in the whole range of $A_{m}$ except the deviation around $30 \mathrm{mG}$. All samples show this deviation but at lower $A_{m}$ as doping percentage is increased. Peak position $H_{m}$, as a function of $A_{m}$ is shown in figure $2 . H_{m}$ shifts towards higher field with increasing $A_{m}$. The behaviour of the amount of trapped flux (area enclosed by the hysteresis loop $\Delta A$ ) with $A_{m}$ is quite interesting. The variation of $\Delta A$ for all samples as a function of $\Delta A$ is shown in figure 3. Initially $\Delta A$ increases with increasing $A_{m}$ except in a certain range in which $\Delta A$ decreases with increasing $A_{m} . A_{m}$ at which $\Delta A$ starts to decrease shifts towards lower value as doping percentage of $\mathrm{Hf}$ is increased. The range of $A_{m}$ in which $\Delta A$ decreases with increasing $A_{m}$ also decreases with increasing percentage of $\mathrm{Hf}$ doping. The first discontinuity in $h_{p}$ vs $A_{m}$ and $\Delta A$ vs $A_{m}$ curves shows interesting correlation.

\section{Discussions}

The modulation amplitude dependence of LFMAS indicates two regions of linear dependence separated by a nonlinear region. The anomalous nonlinear behaviour can be attributed to two different contributions to LFMAS. One of the contributions seems to saturate at low modulation amplitude $\sim 30 \mathrm{mG}$. It has been suggested by Rakvin et al (1989) that the low modulation region results due to a dispersion contribution and the two different contributions to LFMAS comes from two different types of fluxons. Alternatively it has been proposed that the LFMAS is due to two 
components, one of which is due to the modulation of the magnetic flux through the sample and the other due to the components which depend on the boundary current (Pozek et al 1989). However, no definite explanation is available in literature for the anomalous nonlinear dependence on modulation amplitude of LFMAS and there is need for further theoretical efforts.

\section{Acknowledgements}

Financial support from the Department of Science and Technology, New Delhi under the National Programme on superconductivity and the Council of Scientific and Industrial Research, New Delhi is gratefully acknowledged. We are also thankful to Mr D K Kanaujia for technical help.

\section{References}

Blazey K W, Muller K A, Bednorz J G, Berlinger W, Ammoretti G, Buluggiu E, Vera A and Matacotta F C 1987 Phys. Rev. B36 724

Jayaram B, Agarwal S K, Nagpal K C, Gupta A and Narlikar A V 1988 Mater. Res, Bull. 23701

Khachaturyan K, Weber E R, Tejedar P, Stacy A M and Portis A M 1987 Phys. Rev. B36 8309

Müller K A, Takashige M and Bednorz J G 1987 Phys. Rev. Lett. 581143

Portis A M, Blazey K W, Mûller K A and Bednorz J G 1988 Europhys. Lett. 5467

Pozek M, Dulcic A and Rakvin B 1989 Solid State Commun. 70889

Rakvin B, Pozek M, Paljevic M and Brnicevic N 1989 Solid State Commun. 70729

Yashurun Y and Malozemoff A P 1988 Phys. Rev. Lett. 602202 